\title{
Asymptomatic central line-associated bloodstream infections in children implanted with long term indwelling central venous catheters in a teaching hospital, Sri Lanka
}

\author{
J. A. A. S. Jayaweera ${ }^{1,2^{*}}$ (D) and D. Sivakumar ${ }^{1}$
}

\begin{abstract}
Background: Indwelling central venous catheters (CVC) are used to provide long term hemodialysis. The commonest and the severe complication of CVC is the central line-associated bloodstream infection (CLABSI). This study was done to assess the etiology and infectious complications of CVC in children on long term hemodialysis.

Methods: Children newly undergoing hemodialysis and having indwelling CVC were included. They were followed up to a period of 2-years to assess infectious complications. Catheter bundle care approach was employed to prevent infections and other complications. Automated culture from the central catheter and peripheral vein and 2D echocardiography were done in each hemodialysis. Serial procalcitonin (PCT) was measured. Differential time of positivity (DTP) was used to detect CLABSI. During homestay in weekly telephone conversations were done to assess features of infection, and whenever having, we have asked to admit to the tertiary care unit. Logistic regression was performed, and the significant outcome variable was considered following multivariable analysis as a risk factor.

Results: Blood cultures were positive in 1090 (74.5\%) out of 1462 children. According to DTP, 410 (28\%) were having CLABSI, while 520 (35.6\%) were having bacteremia without CLABSI. Out of 410 CLABSI patients, 79 (19.2\%) were asymptomatic. Coagulase-negative Staphylococcus spp. (CoNS) bacteremia was significantly associated with asymptomatic CLABSI. Right-sided infective endocarditis (RS-IE) was significantly associated with asymptomatic CLABSI and asymptomatic bacteremia without CLABSI. CoNS was associated significantly in RS-IE following asymptomatic CLABSI and asymptomatic bacteremia. PCT was in asymptomatic CLABSI was $1.8 \pm 0.9 \mathrm{ng} / \mathrm{mL}$ while in symptomatic CLABSI was $11.3 \pm 2.5 \mathrm{ng} / \mathrm{ml}(P=0.02)$. CoNS bloodstream infection, tunneled $C V C$, peripherally inserted central catheter, femoral site, the number of line days $>90$, receipt of vancomycin, meropenem, or linezolid in the 5 days before CLABSI diagnosis and recurrent bacteremia were risk factors for asymptomatic CLABSI.

(Continued on next page)
\end{abstract}

\footnotetext{
*Correspondence: jaas071@gmail.com; jaas820703@yahoo.com; jaasjayaweera@rit.med.ac.lk

'Department of Microbiology, Faculty of Medicine and Allied Sciences, Rajarata University of Sri Lanka, Microbiology, Saliyapura, Sri Lanka

${ }^{2}$ Teaching Hospital Kandy, Kandy, Sri Lanka
}

(c) The Author(s). 2020 Open Access This article is licensed under a Creative Commons Attribution 4.0 International License, which permits use, sharing, adaptation, distribution and reproduction in any medium or format, as long as you give appropriate credit to the original author(s) and the source, provide a link to the Creative Commons licence, and indicate if changes were made. The images or other third party material in this article are included in the article's Creative Commons licence, unless indicated otherwise in a credit line to the material. If material is not included in the article's Creative Commons licence and your intended use is not permitted by statutory regulation or exceeds the permitted use, you will need to obtain permission directly from the copyright holder. To view a copy of this licence, visit http://creativecommons.org/licenses/by/4.0/ The Creative Commons Public Domain Dedication waiver (http://creativecommons.org/publicdomain/zero/1.0/) applies to the data made available in this article, unless otherwise stated in a credit line to the data. 


\begin{abstract}
(Continued from previous page)
Conclusions: Asymptomatic CLABSI could be a rare occurrence. CoNS was predominantly isolated in patients with asymptomatic CLABSI. RS- IE is a well-known complication in long term indwelling CVC. CoNS was significantly associated with RS-IE following asymptomatic CLABSI. Regular procalcitonin, microbiological, and imaging studies would be essential to detect infectious complications in both symptomatic and asymptomatic patients implanted with long term indwelling CVCs.
\end{abstract}

Keywords: Healthcare-associated infections, Central line-associated bloodstream infection, Asymptomatic bacteremia, Staphylococcus aureus, MRSA, Coagulase-negative Staphylococcus sp., And right sided-infective endocarditis

\section{Background}

Long-term central venous catheter (CVC) is an invasive device that used in children with chronic renal failure. The device resides in a large central vein, usually the superior vena cava. CVC is used for the administration of fluids, medications, blood products, collection of blood, and hemodialysis (HD) [1]. The most common and severe complication associated with $\mathrm{CVC}$ is central lineassociated bloodstream infection (CLABSI) [1, 2].

CLABSI rates vary widely, and infection rates depend on device type and patient population [2]. A study conducted in a pediatric intensive care unit (PICU) in United State of America (USA) from 2006 to 2007 reported that incidence of CLABSI was 3.1 per 1000 central line-days [3]. Another study revealed that incidence was 4.1 per 1.000 central linedays in third world countries [4]. Children with haemopoietic stem cell transplantation, prevalence of CLABSI was 5.3 per 1000 central-line days and most commonly identified organism was Staphylococcus epidermidis [5].

The etiology and the incidence of Chronic Kidney Disease (CKD) could vary with the age [6]. The prevalence of CKD stage II or lower in children is approximately 18.558.3 per million [7]. Compared to adults, in children CKD prevalence is much less but underreporting would mask the true prevalence [8]. In children, structural defects and obstructive uropathy are common before age 5 while hereditary and acquired kidney diseases are common in 5 to 15 years. CKD requires therapeutic measures [9]. When the glomerular filtration rate is below $15 \mathrm{ml} / \mathrm{min} / 1.73 \mathrm{~m}^{2}$, renal replacement therapy such as peritoneal dialysis, hemodialysis, or kidney transplantation is indicated [10].

Bacteremia can occur spontaneously following tissue infection, wound care, surgical procedure, and the use of indwelling intravascular catheters [11]. Bacteremia can be symptomatic or asymptomatic. Bacteremia would lead to develop deep seated abscesses, pneumonia, meningitis, and infective endocarditis especially in patients with valvular heart abnormalities [12]. Transient bacteremia is often asymptomatic, but occasionally can develop fever [13]. The development of symptoms usually suggests more severe infections and risk for sepsis or septic shock would be high [11, 14]. CLABSI often leads to continuous bacteremia. This study was conducted to assess the etiology and infectious complications of central lineassociated bloodstream infections (CLABSI) in children on long term HD.

\section{Methods}

This was a follow up study. The study was conducted at a pediatric unit in a tertiary care hospital, Sri Lanka, from January-2014 to December-2016. Children (2 years to 12 years) who are newly undergoing hemodialysis and having an indwelling central venous line were included and followed up. They were followed up to 2 years to assess any of the infectious complications. Since in all the patients HD was newly initiated, they were kept $>48 \mathrm{~h}$ in the unit. Once patient becomes stable (creatinine decline and reaches a steady level) and no sign of infection they were discharged. We have screened occurrence of CLABSI following after $48 \mathrm{~h}$ of insertion of CVC.

Catheter bundle care approach was employed at the time of insertion and maintenance to prevent infections and other complications [15]. In each time before initiating HD, blood (includes asymptomatic patients) was taken from the central line and peripheral site for culture and antimicrobial susceptibility testing (AST). Also, when the patient presented with fever, blood was taken from the central line and peripheral site for culture and antimicrobial susceptibility testing (AST). Every occasion, the same volume of blood was collected under sterile conditions, and BACTEC semi-automated flat form was used for culture. Differential time positivity (DTP) was used to detect CLABSI, bacteremia, central line colonization, and contamination $[15,16]$. At day $3(72 \mathrm{~h}$ following initial blood culture), a peripheral blood culture was done to assess the clearance following Staphylococcus aureus bacteremia.

Gram staining was performed from all flagged positive BACTEC bottles and inoculated onto blood $\left(10 \% \mathrm{CO}_{2}\right)$, MacConkey (room air), and chocolate agar $\left(10 \% \mathrm{CO}_{2}\right)$ to detect the microbial etiology. The inoculated plates were incubated at $35^{\circ} \mathrm{C}$ in $10 \% \mathrm{CO}_{2}$ to enable bacterial colonies to develop [17]. Positive culture isolates were identified using appropriate identification methods, including morphology of colonies, Gram-stain, and an inhouse set of biochemical tests and further confirmed 
using Rapid 20 E (Enterobacteriaceae), NE (Non-Enterobacteriaceae) and $\mathrm{S}$ (Staphylococcus) semi-automated identification system.

Serial procalcitonin (PCT) and C-reactive protein (CRP) were done to assess the clinical status and the response to the antimicrobial treatment. Also, 2D echocardiography was done in every patient, and in patients with endocarditis serially, it was done to assess the progress/ clinical response. During homestay in weekly telephone conversations were done to assess infectious complications and, if present, asked to admit as soon as possible to the tertiary care facility.

\section{Definitions}

\section{CLABSI}

The Centers for Disease Control and Prevention (CDC) definitions were used to diagnose CLABSI [18].

\section{Bacteremia without line infection}

The quantitative blood cultures were obtained from the CVC and peripheral vein in same time and isolation of the same pathogen from both cultures with time to positive culture $(<2 \mathrm{~h})$ in the CVC and peripheral sample $[15,16]$.

\section{Central line colonizer}

Instances where CVC blood culture is positive but the percutaneous blood culture remained negative indicate colonization of the catheter rather than CRBSI. This is especially applicable to organisms like gram-negative rod or enterococcus $[15,16]$.

\section{Asymptomatic bacteremia}

Instances where the two peripheral blood culture were remained positive for same organism but subject remains without having fever or any other sign/symptom [17].

\section{Exit site infection}

Signs of inflammation is limited to catheter exit site (typically $<2 \mathrm{~cm}$ ) and having a wound swab/ wound secretion culture positivity $[17,18]$.

\section{Tunnel infection}

Inflammation extending more than $2 \mathrm{~cm}$ from exit site and associated with pain and tenderness along the subcutaneous track and having a wound swab/ wound secretion culture positivity [18].

\section{Antimicrobial susceptibility testing}

The antimicrobial susceptibility testing was performed by the disc diffusion test based on Clinical and Laboratory Standards Institute (CLSI) guidelines (M100s27) [19]. The following antimicrobial agents were tested: ampicillin $(30 \mu \mathrm{g})$, amikacin $(30 \mu \mathrm{g})$, Ciprofloxacin $(5 \mu \mathrm{g})$, levofloxacin $(5 \mu \mathrm{g})$, Trimethoprim/ sulfamethoxazole $(1.25 / 23.25 \mu \mathrm{g})$, gentamicin $(10 \mu \mathrm{g})$, Vancomycin $(30 \mu \mathrm{g})$ and Linezolid $(30 \mu \mathrm{g})$.

\section{Statistical analysis}

Data obtained were double entered into a spreadsheet database prepared with Microsoft Excel and compared and cleaned for wrong entries. Statistical analysis was done using SAS version 9.1 [20]. The association of each of the categorical variables with the response variable was assessed by Chi-square test. Further, logistic regression was performed, and variables showing statistically significant $(p<0.05)$ association in univariate analysis with the outcome variable were considered following multivariate analysis as a risk factor. Further, 2 wayANOVA was performed to assess the significance of PCT between symptomatic and asymptomatic CLABSI. Continuous variables were expressed as a measure of central tendency.

\section{Results}

Over the 3 years, 1462 children (2 to 12 years of age) who are newly undergoing long-term HD were included. The mean age of the participant was $7.82 \pm 2.62$ years. Children with congenital malformations and obstructive uropathy requiring HD was significant among children below the age of 5 years $(n=642$, mean age $2.82 \pm 1.43$ years, $p=0.03$ ) while hereditary kidney diseases requiring HD was significant in the age group of 5 to 12 years $(n=822$, mean age $8.82 \pm 1.22, p=0.01)$.

\section{Blood culture results}

Blood cultures were positive in 1090 (74.5\%) out of 1462 children on long term HD. According to differential time positivity, 410 (28\%) were having CLABSI while $520(35.6 \%)$ were having bacteremia without CLABSI, and 160 (11\%) were having central venous line colonizers. Remaining 372 (25.4\%) was culture negative.

\section{CLABSI}

Out of 410 CLABSI patients 331 (80.7\%) were symptomatic. They were presented with fever $(n=331)$, malaise $(n=$ $331)$, and $58(17.5 \%)$ were septic (fever, tachypnoea, tachycardic, PCT $>0.5$ with serum lactate $>4 \mathrm{mmol} / \mathrm{L}$ ) on admission. Methicillin sensitive Staphylococcus aureus (MSSA) was the commonest etiology in symptomatic CLABSI and it was isolated in $212(64.7 \%, p=0.02)$ patients. Candida albicans $(n=17,4.5 \%)$ C. parapsilosis $(n=$ 10, 3.1\%) and Gram negatives [multi-drug resistant Klebsiella pneumoniae $(n=10,3.1 \%)$ Escherichia coli $(n=6,1.5 \%)$ and Citrobacter frundii $(n=3,0.75 \%)]$ were also isolated.

Out of 410 CLABSI patients 79 (19.3\%) were asymptomatic. Coagulase negative staphylococcus sp. [S. epidermidis ( $n=54,68.4 \%), S$. hemolyticus $(n=6,7.7 \%), S$. schleiferi $(n=5,6.4 \%)$, S. lugdunensis $(n=5,6.4 \%)(p=$ 
0.03)] was the commonest etiology for asymptomatic CLABSI (Fig. 1).

Recurrent bacteremia was detected in both symptomatic $(n=98,23.9 \%, P=0.02)$ and asymptomatic CLABSI groups while it was significant in the former. MSSA was the most frequent etiology in recurrent symptomatic CLABSI, and it was isolated in $78(79.5 \%, p=0.01)$ patients.

\section{Bacteremia without CLABSI}

Out of 520 bacteremic patients 398 (76.5\%) were symptomatic. They were presented with fever $(n=398)$, malaise $(n=398)$, cough $(n=90)$, difficulty in breathing $(n=$ $88)$, dysuria $(n=48)$, backache $(n=22)$, acute abdomen $(n=10)$, alteration of consciousness $(n=8)$, and 68 (17.1\%) were septic (fever, tachypnoea, tachycardic, PCT $>0.5$ with serum lactate $>4 \mathrm{mmol} / \mathrm{L})$ on admission. Methicillin sensitive $S$. aureus (MSSA) was the commonest etiology and was isolated in $245(61.5 \%, p=0.03)$ patients. Methicillin resistant $S$. aureus (MRSA) in 42 $(10.5 \%)$ patients while coagulase negative staphylococcus sp.(45, 11.3\%) [S. epidermidis $(n=27,6.8 \%)$, S. hemolyticus $(n=18,4.5 \%))]$, E. fecalis $(n=8,2 \%)$, E. faceum $(n=$ 4, $1 \%)$, Candida albicans $(n=8,2 \%)$ C. parapsilosis $(n=$ $8,2 \%)$, C. tropicalis $(n=7,2 \%)$ and $K$. pneumoniae ( $n=$ $10,2.5 \%)$ E. coli $(n=16,4 \%)$, Pseudomonas aeruginosa $(n=8,4 \%)$, and C. frundii $(n=6,3 \%)$ was also isolated.

Out of 520 bacteremic patients 122 (23.4\%) were asymptomatic. Coagulase negative staphylococcus sp. [S. epidermidis $(n=86,70.5 \%), S$. hemolyticus $(n=14,11.4 \%), S$. schleiferi $(n=6,5 \%), S$. lugdunensis $(n=3,2.5 \%)(p=$ $0.01)$ ] was the commonest etiology for asymptomatic bacteremic and C. parapsilosis $(n=3,2.5 \%), C$. albicans $(n=3,2.5 \%)$ MSSA $(n=3,2.5 \%)$ MRSA $(1,0.8 \%)$, Escherichia coli $(n=3,2.5 \%)$ was also isolated (Fig. 2$)$.
Recurrent bacteremia was detected in both symptomatic $(n=78,19.6 \%, P=0.04)$ and asymptomatic groups while it was significant in the former. MSSA was the most frequent etiology in recurrent symptomatic bacteremia, and it was isolated in $58(74.3 \%, p=0.03)$ patients.

\section{Venous catheter colonizer (VCC)}

Out of 160 VCC, coagulase negative Staphylococcus sp. [S. epidermidis $(n=86,53.7 \%)$, S. hemolyticus $(n=16$, $10 \%)$, S. schleiferi $(n=8,5 \%)$, S. lugdunensis $(n=4,2.5 \%)$ $(p=0.01)]$ was the commonest while C. parapsilosis $(n=$ $12,7.5 \%), C$. albicans $(n=8,5 \%)$, MSSA $(n=8,5 \%)$ MRSA (4,2.5\%), Escherichia coli $(n=4,2.5 \%)$, Acinetobacter bahumanii $(n=4,2.5 \%), C$. frundii $(n=4,2.5 \%)$ and $P$. aeruginosa $(n=4,2.5 \%)$ was also isolated.

In all patients with bacteremia, procalcitonin was elevated $(>0.5 \mathrm{ng} / \mathrm{ml})$. PCT was in asymptomatic CLABSI was $1.8 \pm 0.9$ while in symptomatic CLABSI was $11.3 \pm$ $2.5(p=0.01)$.

\section{Antimicrobial susceptibility pattern of isolated microbes from asymptomatic and symptomatic CLABSI}

Compared to S. epidermidis isolates following symptomatic CLABSI, S. epidermidis isolates following asymptomatic CLABSI were having significant resistance to oxacillin, cefoxitin, ciprofloxacin, levofloxacin, Trimethoprim/ sulfamethoxazole, Amikacin, gentamicin, erythromycin, and clindamycin while all were susceptible to vancomycin, linezolid, and tigecycline (Table 1).

\section{Other infective complications}

Central venous catheter exit site $(n=98,6.7 \%)$ and tunnel infections $(n=70,4.8 \%)$ were detected in long term indwelling venous catheters. Exit site infection was observed $7.5 \pm 6.25$ months, and tunnel infection was observed $11 \pm$

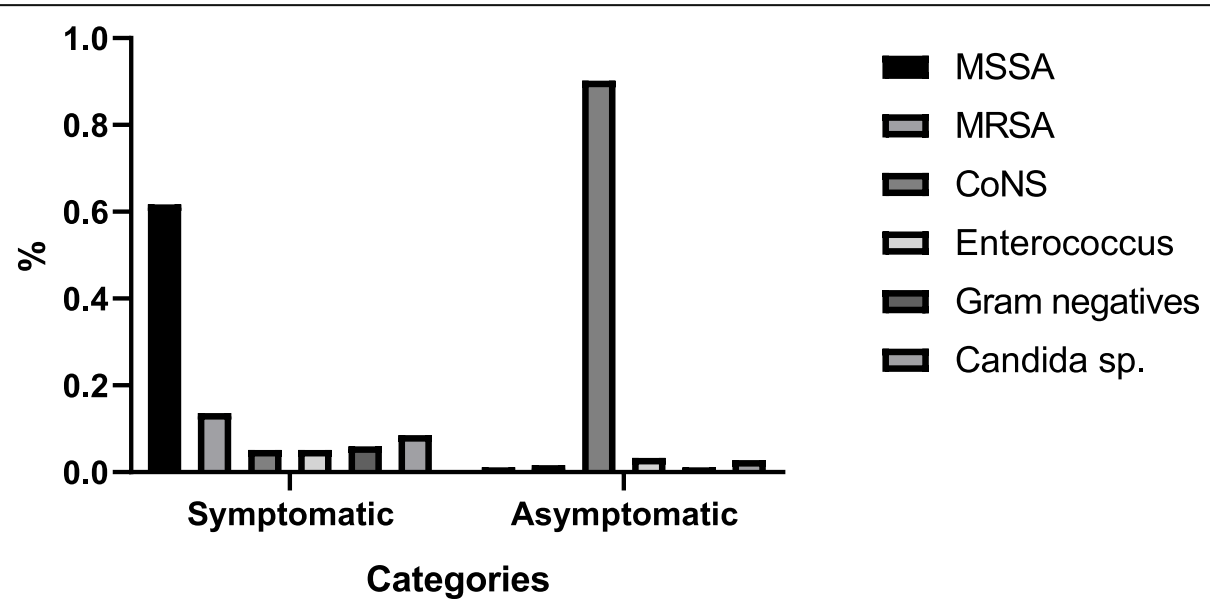

Fig. 1 Microbial etiology in symptomatic and symptomatic patients with central line-associated bloodstream infections. MSSA- Methicillinsensitive S. aureus, MRSA- Methicillin-resistant S. aureus, CoNS- Coagulase-negative Staphylococcus sp. 


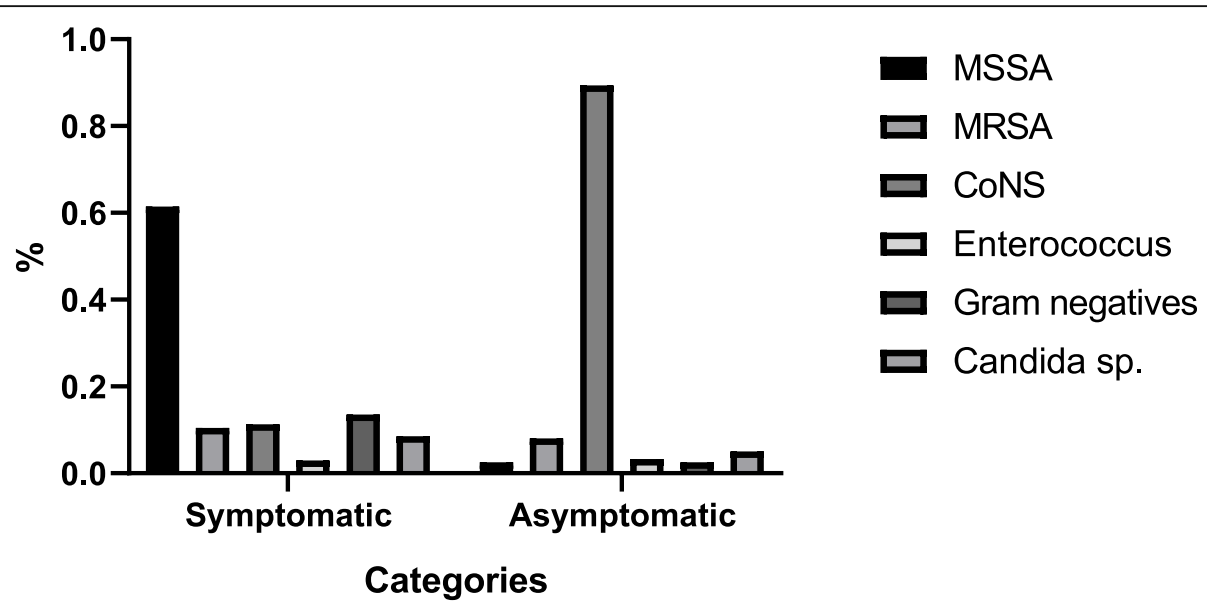

Fig. 2 Microbial etiology in symptomatic and symptomatic bacteremic patients. MSSA- Methicillin-sensitive S. aureus, MRSA- Methicillin-resistant S. aureus, CoNS- Coagulase-negative Staphylococcus sp.

2.75 months following the insertion of a venous catheter. Further, venous catheter blockage was detected in 88 (6\%), and in all, including exit and tunnel infections catheter, revisal was done to minimize systemic infections. The blockage was observed $0.82 \pm 0.43$ years following the insertion of a central venous catheter.

Right sided infective endocarditis (IE) was detected in $122(8.3 \%)$ patients and was observed $8.6 \pm 3$ months following insertion of venous catheter. It was detected in CLABSI symptomatic $(n=29,24.5 \%)$, CLABSI asymptomatic $(n=42,34.5 \%)$ ' symptomatic $(n=14,11.5 \%)$ and asymptomatic bacteremic ( $n=36,29.5 \%)$ patients and is significant in asymptomatic CLABSI and asymptomatic bacteremic patients (Table 2).

Further, catheter tip endocarditis $(n=88,6 \%)$ and left sided IE $(n=31,2.1 \%)$ was detected in all above mentioned groups. Catheter tip endocarditis was observed $6.25 \pm 2.9$ months following insertion of venous catheter and it was significantly detected in asymptomatic bacteremic (without CLABSI) $(n=48,54.5 \%, p=0.03)$ and non bacteremic children $(n=39,44.3 \%, p=0.04)$. Coagulase negative Staphylococcus sp.[s. epidermidis

Table 1 Antimicrobial susceptibility profile of coagulase-negative Staphylococcus sp. (CoNS) isolates in asymptomatic and symptomatic central line-associated bloodstream infections

\begin{tabular}{|c|c|c|c|c|c|c|c|}
\hline \multirow[t]{3}{*}{ Antibiotic } & \multicolumn{6}{|l|}{ CLABSI } & \multirow{3}{*}{$\begin{array}{l}P \\
\text { value }\end{array}$} \\
\hline & \multicolumn{2}{|c|}{ Symptomatic- CoNS } & \multicolumn{4}{|c|}{ Asymptomatic- CoNS } & \\
\hline & $\begin{array}{l}\text { S. epidermidis } \\
(\boldsymbol{n}=10)\end{array}$ & $\begin{array}{l}\text { S. hemolyticus } \\
(\boldsymbol{n}=6)\end{array}$ & $\begin{array}{l}\text { S. epidermidis } \\
(\boldsymbol{n}=54)\end{array}$ & $\begin{array}{l}\text { S. hemolyticus } \\
(\boldsymbol{n}=6)\end{array}$ & $\begin{array}{l}\text { S. schleiferi } \\
(\boldsymbol{n}=5)\end{array}$ & $\begin{array}{l}\text { S. lugdunensis } \\
(\boldsymbol{n}=5)\end{array}$ & \\
\hline Ampicillin $(30 \mu \mathrm{g})$ & $2(20 \%)$ & $1(17 \%)$ & $47(87.3 \%)^{*}$ & $6(100 \%)$ & $5(100 \%)$ & $5(100 \%)$ & $0.03^{*}$ \\
\hline Cefoxitin $(30 \mu \mathrm{g})$ & $2(20 \%)$ & $1(17 \%)$ & $45(85.7 \%)^{* *}$ & $6(100 \%)$ & $5(100 \%)$ & $5(100 \%)$ & $0.02^{* *}$ \\
\hline Oxacillin & $2(20 \%)$ & $1(17 \%)$ & $45(85.7 \%)^{* * *}$ & $6(100 \%)$ & $5(100 \%)$ & $5(100 \%)$ & $0.03^{* * *}$ \\
\hline Ciprofloxacin $(5 \mu \mathrm{g})$ & $1(10 \%)$ & $1(17 \%)$ & $45(85.7 \%)^{a}$ & $4(67 \%)$ & $5(100 \%)$ & $5(100 \%)$ & $0.03^{a}$ \\
\hline Levofloxacin $(5 \mu \mathrm{g})$ & $2(20 \%)$ & $1(17 \%)$ & $45(85.7 \%)^{\boldsymbol{\mu}}$ & $4(67 \%)$ & $4(80 \%)$ & $5(100 \%)$ & $0.03^{\mu}$ \\
\hline $\begin{array}{l}\text { Trimethoprim/ sulfamethoxazole } \\
(1.25 / 23.25 \mu \mathrm{g})\end{array}$ & $1(10 \%)$ & $1(17 \%)$ & $42(79.3 \%)$ & $4(67 \%)$ & $5(100 \%)$ & $5(100 \%)$ & 0.03 \\
\hline Amikacin $(30 \mu g)$ & $3(30)$ & $1(17 \%)$ & $42(79.3 \%)$ & $4(67 \%)$ & $5(100 \%)$ & $5(100 \%)$ & 0.03 \\
\hline Gentamicin $(10 \mu \mathrm{g})$ & $2(20)$ & $1(17 \%)$ & $42(79.3 \%)$ & $4(67 \%)$ & $5(100 \%)$ & $5(100 \%)$ & 0.03 \\
\hline Erythromycin $(30 \mu \mathrm{g})$ & $2(20)$ & $1(17 \%)$ & $42(79.3 \%)$ & $4(67 \%)$ & $5(100 \%)$ & $5(100 \%)$ & 0.03 \\
\hline Clindamycin $(30 \mu \mathrm{g})$ & $2(20)$ & $1(17 \%)$ & $42(79.3 \%)$ & $4(67 \%)$ & $5(100 \%)$ & $5(100 \%)$ & 0.03 \\
\hline vancomycin & 0 & 0 & 0 & 0 & 0 & 0 & - \\
\hline Linezolid & 0 & 0 & 0 & 0 & 0 & 0 & - \\
\hline Tigecycline & 0 & 0 & 0 & 0 & 0 & 0 & - \\
\hline
\end{tabular}

$P<0.05$ taken as significant

* Ampicillin resistance is significantly low in S. epidermidis; ${ }^{* *}$ Cefoxitin resistance is significantly low in S. epidermidis; ${ }^{* *}$ Oxacillin resistance is significantly low in S. epidermidis; a Ciprofloxacin resistance is significantly low in S. epidermidis; $\mu$ Levofloxacin resistance is significantly low in S. epidermidis 
Table 2 The microbial etiology for right-sided infective endocarditis in symptomatic and asymptomatic central line-associated bloodstream infections and bacteremia without central line infection patients

\begin{tabular}{|c|c|c|c|c|c|}
\hline \multirow{3}{*}{$\begin{array}{l}\text { Isolated } \\
\text { microbes }\end{array}$} & \multicolumn{4}{|c|}{ Right sided infective endocarditis ( $\boldsymbol{n}=122,8.3 \%)$} & \multirow{3}{*}{$\begin{array}{l}\boldsymbol{P} \\
\text { value } \\
0.03\end{array}$} \\
\hline & \multicolumn{2}{|l|}{ CLABSI $(72,59 \%)$} & \multicolumn{2}{|c|}{ Bacteremia without CLABSI $(50,41 \%)$} & \\
\hline & Symptomatic $(\boldsymbol{n}=30,24.5 \%)$ & Asymptomatic $(\boldsymbol{n}=42,34.5 \%)$ & Symptomatic $(\boldsymbol{n}=14,11.5 \%)$ & Asymptomatic $(\boldsymbol{n}=36,29.5 \%)$ & \\
\hline MSSA & $12^{* *}$ & $13^{* *}$ & 4 & 6 & $0.03^{*}$ \\
\hline MRSA & $12^{£}$ & $13^{ \pm}$ & $7^{£}$ & 7 & $0.02^{\mathrm{f}}$ \\
\hline CoNS & 2 & $14^{a}$ & 1 & $16^{a}$ & $0.02^{a}$ \\
\hline S. epidermidis & 1 & 6 & - & 9 & 0.07 \\
\hline S. hemolyticus & 1 & 1 & 1 & 2 & 0.08 \\
\hline S. schleiferi & - & 1 & - & 4 & 0.07 \\
\hline S. lugdunensis & - & 1 & - & 1 & 0.07 \\
\hline Enterococcus sp. & 2 & 1 & - & 1 & 0.08 \\
\hline C. albicans & 3 & 1 & 1 & 2 & 0.07 \\
\hline C. parapsilosis & 1 & 1 & 1 & 4 & 0.08 \\
\hline
\end{tabular}

CLABSI central line-associated bloodstream infections, MSSA Methicillin-sensitive S. aureus, MRSA Methicillin-resistant S. aureus, CoNS Coagulase-negative Staphylococcus $s p$.

$P<0.05$ taken as significant

${ }^{* *}$ MSSA was associated significantly in symptomatic and asymptomatic CLABSI, ${ }^{\mathrm{E}}$ MRSA was associated significantly in right sided IE following symptomatic, asymptomatic CLABSI and symptomatic bacteremic without CLABSI and ${ }^{\circ}$ CoNS was associated significantly in right sided IE following asymptomatic CLABSI and asymptomatic bacteremic without CLABSI

( $n=18,37.5 \%)$, S. hemolyticus $(n=12,25 \%)$, S. schleiferi $(n=6,12.5 \%), S$. lugdunensis $(n=3,6.25 \%)(p=0.01)]$ was the commonest while $C$. parapsilosis $(n=4,8.3 \%)$, C. albicans $(n=3,6.25 \%)$, MSSA $(n=1,1.1 \%)$ and MRSA $(n=1,1.1 \%)$ also isolated from the blood cultures.

Patients with left sided IE was observed $0.42 \pm 0.34$ years following insertion of venous catheter and it was detected in symptomatic bacteremic $(n=19,61.2 \%, p=$ $0.03)$ and symptomatic CLABSI children $(n=12,38.7 \%$, $p=0.04)$. MRSA in $12(38.7 \%)$, MSSA $(n=10,34 \%), E$. fecalis $(n=3,10 \%)$, E. faceum $(n=3,10 \%)$, C. albicans $(n=2,6.7 \%)$ and $C$. parapsilosis $(n=1,3.3 \%)$ was detected in patients with left sided IE.

Following univariate analysis, coagulase-negative Staphylococcus sp., tunneled central venous catheter, PICC, femoral site, a number of line-days $>90$, receipt of vancomycin, meropenem or linezolid in the 5 days before obtaining blood cultures and recurrent bacteremia was significantly associated with increased risk of asymptomatic CLABSI. Further, receipt of vancomycin, meropenem, or linezolid in the 5 days before obtaining blood cultures was significantly associated with non-bacteremia (Table 3).

According to multivariate analysis coagulase negative staphylococcus sp. blood stream infection (OR: 7.6, 95\% CI: 6.5-8.3, $P=0.03$ ), having tunneled CVC (OR: 4.4, 95\% CI: 3.8-4.9, $P=0.03)$, peripherally inserted central catheter (OR: 4.9, 95\% CI: 3.9-6.2, $P=0.02$ ), femoral site (OR: 3.7, 95\% CI: 2.8-4.3, $P=0.01$ ), number of line days $>90$ (OR: 2.1, 95\% CI: 1.3-4.0, $P=0.02$ ), receipt of vancomycin, meropenem or linezolid in the 5 days prior to CLABSI diagnosis (OR: 2.1, 95\% CI: 1.3-4.0, $P=0.02$ ) and recurrent bacteremia (OR: 8.7, 95\% CI: 7.8-9.3, $P=$ 0.01 ) were associated with asymptomatic CLABSI in patients with long term hemodialysis (Table 4).

\section{Discussion}

CVCs are not without risk and following placement it can develop multiple complications. Significant morbidity, mortality can result following complications and can cause a significant burden leading to high expenditure, prolonged hospitalization, and poor quality of life [21-23].

To our knowledge, this is the first report of asymptomatic CLABSI in patients who are undergoing long term HD. Transient asymptomatic bacteremia is a known phenomenon, while CLABSI is often symptomatic and followed with continuous bacteremia $[5,6]$. Contrary, asymptomatic CLABSI could be a rare occurrence, and we were able to explore it following screening blood cultures in every patient before each HD. CoNS was predominantly isolated in patients with asymptomatic CLABSI, while MSSA was predominantly isolated in patients with symptomatic CLABSI. Zierdt in 1983 has described that the intermittent or transient or asymptomatic $S$. epidermidis bacteremia can frequently occur inpatients and as well as in healthy humans [24].

Right-sided IE is a well-known complication following long term indwelling CVCs [15]. Often is diagnosed with screening echocardiography following MSSA, MRSA bacteremia or candidemia, and instances where having an inadequate response following appropriate antimicrobials in other bacteremia (CoNS, Enterococcus sp., any of 
Table 3 Univariate analysis of risk factors for asymptomatic central line-associated bloodstream infections in children on hemodialysis

\begin{tabular}{|c|c|c|c|}
\hline \multirow[t]{2}{*}{ Variables } & \multicolumn{2}{|l|}{ CLABSI $(\boldsymbol{n}=410)$} & \multirow{2}{*}{$\begin{array}{l}\text { Non- } \\
\text { bacteremics } \\
\text { Odds ratio } \\
(95 \% \mathrm{Cl})\end{array}$} \\
\hline & $\begin{array}{l}\text { Symptomatic } \\
\text { Odds ratio (95\% } \\
\text { Cl) }\end{array}$ & $\begin{array}{l}\text { Asymptomatic } \\
\text { Odds ratio (95\% } \\
\text { Cl) }\end{array}$ & \\
\hline \multicolumn{4}{|l|}{ Microbial etiology } \\
\hline MSSA & $2.2(1.1-3.9)$ & & \\
\hline MRSA & $2.4(1.9-3.1)$ & & \\
\hline CoNS & & $3.4(2.9-3.8)$ & \\
\hline Female sex & - & - & \\
\hline \multicolumn{4}{|l|}{ Type of CVC } \\
\hline Tunneled & & $2.7(1.8-4.3)$ & \\
\hline Non-tunneled & - & - & - \\
\hline \multicolumn{4}{|l|}{ Site of insertion } \\
\hline Internal jugular & - & - & - \\
\hline PICC & & $2.9(1.9-4.2)$ & \\
\hline Subclavian & - & - & - \\
\hline Femoral & & $2.7(1.8-4.3)$ & \\
\hline Number of line-days $>90$ & & $2.3(1.3-4.0)$ & \\
\hline Duration of hospital stay & - & - & - \\
\hline $\begin{array}{l}\text { Receipt of vancomycin, meropenem or linezolid in the } 5 \text { days prior to obtaining blood } \\
\text { cultures }\end{array}$ & & $3.7(2.8-4.6)$ & $\begin{array}{l}4.7(3.8- \\
5.3)\end{array}$ \\
\hline No. of times intravenous medications given in the prior day (mean \pm SD) & - & - & - \\
\hline Recurrent bacteremia & & $2.7(1.8-4.3)$ & \\
\hline
\end{tabular}

All significant $(P<0.05)$ values are displayed with the odds ratio

CLABSI central line-associated bloodstream infections, MSSA Methicillin-sensitive S. aureus, MRSA Methicillin-resistant S. aureus, CoNS Coagulase-negative Staphylococcus sp., CVC central venous catheter, PICC peripherally inserted central catheter, SD standard deviation and CI confidence interval

significant bacteremia) [22-25]. In here, CoNS was significantly associated with right-sided IE following asymptomatic CLABSI and asymptomatic bacteremia without CLABSI patients. Kendirli et al. in 2017 found that $S$. epidermidis was commonly identified in children with CLABSI [26]. Another study revealed that S. aureus was the most common organism responsible for right sided IE and IE in tricuspid valve develop more frequently in heroin users [27]. Further, MSSA or MRSA, Streptococci spp. or Enterococci spp. is responsible for acute IE [28]. In here, MSSA was associated significantly following right sided-IE in symptomatic and asymptomatic CLABSI patients while MRSA was associated significantly in right-sided IE following symptomatic, asymptomatic CLABSI and symptomatic bacteremia without CLABSI patients. The above scenarios were

Table 4 Multivariate analysis of risk factors for asymptomatic central line-associated bloodstream infections in children on hemodialysis

\begin{tabular}{llr}
\hline Variables & \multicolumn{2}{c}{$\begin{array}{l}\text { Asymptomatic CLABSI } \\
\text { Odds ratio (95\% CI) P value }\end{array}$} \\
\hline CoNS bloodstream infection & $7.6(6.5-8.3)$ & 0.01 \\
Tunneled-CVC & $4.4(3.8-4.9)$ & 0.03 \\
Site of insertion -PICC & $4.9(3.9-6.2)$ & 0.02 \\
Site of insertion -Femoral & $3.7(2.8-4.3)$ \\
Number of line-days $>\mathbf{9 0}$ & $2.1(1.3-4.0)$ & 0.01 \\
Receipt of vancomycin, meropenem or linezolid in the $\mathbf{5}$ days prior to CLABSI diagnosis & $3.7(2.8-4.6)$ & 0.02 \\
Recurrent bacteremia & $8.7(7.8-9.3)$ & 0.01 \\
\hline
\end{tabular}

CLABSI central line-associated bloodstream infections, MSSA Methicillin-sensitive S. aureus, MRSA Methicillin-resistant S. aureus, CoNS Coagulase-negative Staphylococcus sp., CVC central venous catheter, PICC peripherally inserted central catheter and Cl- confidence interval

$P<0.05$ taken as significant 
detected as an outcome of screening blood cultures and echocardiography. Application of such practice in busy and resource-limited clinical setup needs to be validated.

Contrast to $S$. epidermidis following symptomatic CLABSI, S. epidermidis following asymptomatic CLABSI had significant antimicrobial resistance. It could be an outcome following the segregation of multiple genetic segments in microbial DNA. Since bacterial genetic capacity is often constant, this would reduce the virulence capacity. Various studies have shown that the genetic perturbations responsible for antibiotic resistance modulate bacterial biology and fitness [29]. In many cases, measurements of bacterial growth in animal hosts have revealed fitness and virulence attenuations that agree with in vitro tests, leading to the view that pathogens incur fitness trade-offs that compromise their pathogenic potential. Perhaps, the drug resistance increases pathogen fitness during infection [30,31].

Based on multivariate analysis having a CoNS bloodstream infection and tunneled CVC were risk factors for the development of asymptomatic CLABSI in patients with long term HD. Having tunneled CVC is a known risk factor in CLABSI [32]. Further, the peripherally inserted central catheter (PICC) and femoral site were risk factors for the development of asymptomatic CLABSI in patients with long term HD. Both PICC [33] and femoral site [34] is known risk factor in CLABSI. Contrary, no evidence of higher infection risk when the catheter is inserted into the subclavian, jugular, or femoral vein, as demonstrated for adult patients [33]. A number of line days $>90$ was a risk factor for the development of asymptomatic CLABSI in patients with long term HD. The risk of bacteremia is highest in hemodialysis patients using a CVC for vascular access, and increases in a linear fashion with the duration of catheter use [2, 4-8]. Prolonged use of CVC (7 or more days) considered the risk for CLABSI [31]. A study by Costello et al. analyzed 3319 admissions to the pediatric cardiac intensive care unit found that a central venous line in place for $\geq 7$ days [34]. Also, receipt of vancomycin, meropenem, or linezolid in the 5 days before CLABSI diagnosis was a risk factor for the development of asymptomatic CLABSI in patients with long term hemodialysis. Receipt of high-end antibiotics (vancomycin, meropenem, or linezolid) would suppress the symptoms of bacteremia in great. Because the use of such broad-spectrum antibiotics around 5 days would suppress the bacteremia leading state of partially treated [35]. Depending on etiology for bacteremia without detected focus, at least 7-14 days of therapy is recommended [28]. Furthermore, recurrent bacteremia was a risk factor for the development of asymptomatic CLABSI in patients with long term hemodialysis. Recurrent bacteremia is a risk factor for the development of CLABSI [36, 37].
Here, PCT was in asymptomatic CLABSI was $1.8 \pm 0.9$ $\mathrm{ng} / \mathrm{mL}$ while in symptomatic CLABSI was $11.3 \pm 2.5 \mathrm{ng} /$ $\mathrm{mL}$. The procalcitonin cut-off value to detect sepsis was $\geq 0.5 \mathrm{ng} / \mathrm{mL}$, with a corresponding sensitivity of $76 \%$ and specificity of $69 \%$. Different studies done in different settings the procalcitonin had a fair diagnostic accuracy for bacteremia in adult patients suspected of infection or sepsis. Perhaps, low procalcitonin levels can be used to rule out the presence of bacteremia [38, 39].

\section{Limitations}

We have not performed anaerobic blood cultures to detect any of anaerobic etiology. Specific fungal cultures using a semi-automated platform was also not performed. We have used only the DTP method to detect CLABSI. The overall sensitivity and specificity of a DTP of $\geq 120$ min for diagnosing CLABSI were $85 \%$ (95\% confidence interval $[\mathrm{CI}], 74$ to $93 \%)$ and $82 \%(95 \% \mathrm{CI}, 66$ to $92 \%)$, respectively. Further, we have not performed $S$. epidermidis molecular genetics related to antimicrobial resistance. Due to the low number of MSSA and MRSA cases, risk factor analysis was unable to perform related to symptomatic and asymptomatic CLABSI.

\section{Conclusions}

Asymptomatic CLABSI could be a rare occurrence. CoNS was predominantly isolated in patients with asymptomatic CLABSI. Right-sided IE is a well-known complication following long term indwelling central venous catheters. CoNS was significantly associated with right-sided IE following asymptomatic CLABSI and asymptomatic bacteremia without CLABSI patients. Regular procalcitonin, microbiological, and imaging studies will be essential to detect infectious complications in both symptomatic and asymptomatic children implanted with long term indwelling CVCs.

\section{Abbreviations \\ CVC: Central venous catheter; CLABSI: Central line-associated bloodstream in- fection; USA: United State of America; PICC: Peripherally inserted central catheter; HD: Hemodialysis; CoNS: Coagulase-negative Staphylococcus sp.; RS- IE: Right-sided infective endocarditis; CKD: Chronic Kidney disease; DTP: Differential time positivity; PCT: Procalcitonin; MRSA: Methicillin-resistant Staphylococcus aureus; MSSA: Methicillin sensitive Staphylococcus aureus; $\mathrm{Cl}$ : Confidence interval}

\section{Acknowledgments \\ I would like to acknowledge Dr. A. Joseph for providing support during bacterial culture, microbial identification, and antimicrobial susceptibility testing.}

\section{Authors' contributions}

JAASJ designed the study, and all authors participated in data analysis. JAASJ and DS carried out the lab work. JAASJ drafted the manuscript, and the final manuscript was read and approved by both authors.

\section{Funding}

No funding. 


\section{Availability of data and materials}

The datasets used and analyzed in the current study are available from the corresponding author on reasonable request.

\section{Ethics approval and consent to participate}

Ethical approval for all experimental protocol/s was approved by the ethical review and publication committee, Teaching Hospital Kandy, Sri Lanka, and to participate in the study informed written consent obtained from the legal guardians/ parents. Further all experiments were performed in accordance with relevant guidelines and regulations.

\section{Consent for publication}

Not applicable.

\section{Competing interests}

The authors declare that there is no conflict of interest regarding the publication of this article.

Received: 17 February 2020 Accepted: 22 June 2020

Published online: 29 June 2020

\section{References}

1. Centers for Medicare and Medicaid Services (CMS) HAC Posting on Hospital Compare. Baltimore: CMS; 2011. [Accessed 1 July 2019]. http://www.cms. gov/HospitalQualitylnits/06/HACPost.asp.

2. Shelly MA, Concannon C, Dumyati G. Device use ratio measured weekly can reliably estimate central line-days for central line-associated bloodstream infection rates. Infect Control Hosp Epidemiol. 2011;32:727-30.

3. Jackson SS, Leekha S, Magder LS, Pineles L, Anderson DJ, et al. The effect of adding comorbidities to current centers for disease control and prevention central-line-associated bloodstream infection risk-adjustment methodology. Infect Control Hosp Epidemiol. 2017;3:1-6.

4. Rosenthal VD, Ramachandran B, Villamil-Gómez W, Armas-Ruiz A, Navoa-Ng $J A$, et al. Impact of a multidimensional infection control strategy on central line-associated bloodstream infection rates in pediatric intensive care units of five developing countries: findings of the international nosocomial infection control consortium (INICC). Infection. 2012:40:415-23.

5. Cole TS, Rogerson E, Collins J, Galloway A, Clark J. Central venous catheter related blood stream infections in children undergoing hematopoietic stem cell transplant for primary immunedefiency and other nonmalignant disorder. Pediatr Infect Dis J. 2011;30:1098-100.

6. Flynn J, Zhang Y, Solar-Yohay S, Shi V. Clinical and demographic characteristics of children with hypertension. Hypertension. 2012;60(4):104754. https://doi.org/10.1161/HYPERTENSIONAHA.112.197525.

7. Ardissino G, Dacco V, Testa $S$, et al. Epidemiology of chronic renal failure in children: data from the ItalKid project. Pediatrics. 2003;111(4):e382-7.

8. Choi Al, Rodriguez RA, Bacchetti P, Bertenthal D, Hernandez GT, O'Hare AM. White/black racial differences in risk of end-stage renal disease and death. Am J Med 2009; 122(7):672-678.

9. Siriwardhana EARIE, Perera PAJ, Sivakanesan R, Abeysekara T, Nugegoda DB, Jayaweera JAAS. Dehydration and malaria in augmenting the risk of developing chronic kidney disease in Sri Lanka. Indian J Nephrol. 2014;24:16

10. Bush MS. Bacteremia. MSD Manual [Accessed 1 July 2019]. https://www. msdmanuals.com/professional/infectious-diseases/biology-of-infectiousdisease/bacteremia.

11. Gould JM, Rodgers JL. Clinical Syndromes and Cardinal Features of Infectious Diseases: Approach to Diagnosis and Initial Management. Song SS edi. Principles and Practice of Pediatric Infectious Diseases (Fourth Edition), 2012 Elsevier. Philadelphia.

12. Jayaweera JAAS, Reyes MLM. Antimicrobial misuse in pediatric urinary tract infections: recurrences and renal scarring. Ann Clin Microbiol Antimicrob. 2018;17:27. https://doi.org/10.1186/s12941-018-0279-4.

13. Wijesekara PNK, Kumbukgolla WW, Jayaweera JAAS, Rawat D. Review on usage of vancomycin in livestock and humans: maintaining its efficacy, prevention of resistance and alternative therapy. Vet Sci. 2017:4:6.

14. Ray-Barruel G, Xu H, Marsh N, Cooke M, Rickard CM. Effectiveness of insertion and maintenance bundles in preventing peripheral intravenous catheter-related complications and bloodstream infection in hospital patients: a systematic review. Infect Dis Health. 2019:S2468-0451(19):300161. https://doi.org/10.1016/j.idh.2019.03.001.
15. Park K-H, et al. Diagnostic usefulness of differential time to positivity for catheter-related candidemia. J Clin Microbiol. 2014;52:2566. https://doi.org/ 10.1128/JCM.00605-14

16. Negi B, Kumar D, Kumbukgolla W, Jayaweera S, Ponnan P, Singh R, et al. Anti-methicillin resistant Staphylococcus aureus activity, synergism with oxacillin and molecular docking studies of metronidazole-triazole hybrids. Eur J Med Chem. 2016;115:426-37.

17. O'Grady NP, Alexander M, Burns LA, Dellinger EP, Garland J, Heard SO, et al. Healthcare infection control practices advisory committee (HICPAC) (appendix 1). Summary of recommendations: guidelines for the prevention of intravascular catheter-related infections. Clin Infect Dis. 2011;52:1087-99.

18. Bloodstream Infection Event (Central Line-Associated Bloodstream Infection and Non-central Line Associated Bloodstream Infection). CDC. Device associated module. 2020. [Accessed 1 May 2020]. https://www.cdc.gov/ nhsn/PDFs/pscManual/4PSC_CLABScurrent.pdf.

19. Performance Standards for Antimicrobial Disk Susceptibility Tests. Clinical and Laboratory Standards Institute. M02-A11 (Approved Standard-Eleventh Edition); 2016.

20. SAS Institute Inc. Language reference: concepts. 3rd ed. Cary: SAS Institute Inc.; 2005.

21. Jalwal GK, Rajagopalan V, Bindra A, Goyal K, Rath GP, Kumar A, et al. Percutaneous retrieval of malpositioned, kinked and unraveled guide wire under fluoroscopic guidance during central venous cannulation. J Anaesthesiol Clin Pharmacol. 2014;30:582.

22. Kothalawala M, Jayaweera JAAS, Arunan S. Jayathilake JAMA. The emergence of non-albicans candidemia and evaluation of HiChrome Candida differential agar and VITEK2 YST ${ }^{\oplus}$ platform for differentiation of Candida bloodstream isolates in teaching hospital Kandy, Sri Lanka BMC Microbiology 2019; 19:136 https://doi.org/10.1186/s12866-019-1518-3.

23. Jayaweera JA, Kumbukgolla WW. Antibiotic resistance patterns of methicillin-resistant Staphylococcus aureus (MRSA) isolated from livestock and associated farmers in Anuradhapura. Sri Lanka Germs. 2017;7(3):132-9.

24. Zierdt CH. Evidence for transient Staphylococcus epidermidis bacteremia in patients and in healthy humans. J Clin Microbiol. 1983;17(4):628-30.

25. Andersson DI, Hughes D. Antibiotic resistance and its cost: is it possible to reverse resistance? Nat Rev Microbiol. 2010:8:260-71.

26. Kendirli T, Yaman A, Ödek C, Özdemir H, et al. Central line-associated bloodstream infections in pediatric intensive care unit. J Pediatr Emerg Intensive Care Med. 2017:4:42-6.

27. Jain $\mathrm{V}$, Yang MH, Kovacicova-Lezcano G, Juhle LS, Bolger AF, Winston LG. Infective endocarditis in an urban medical center: association of individual drugs with valvular involvement. J Inf Secur. 2008:57:132-8.

28. Forrest GN, Arnold RS, Gammie JS, Gilliam BL. Single center experience of a vancomycin resistant enterococcal endocarditis cohort. J Inf Secur. 2011;63: $420-8$.

29. Roux D, Danilchanka O, Guillard T, et al. Fitness cost of antibiotic susceptibility during bacterial infection. Sci Transl Med. 2015;7:297ra114.

30. Skurnik D, Roux D, Cattoir V, et al. Enhanced in vivo fitness of carbapenemresistant oprD mutants of Pseudomonas aeruginosa revealed through highthroughput sequencing. Proc Natl Acad Sci U S A. 2013;110:20747-52.

31. Marik PE, Flemmer M, Harrison W. The risk of catheter-related bloodstream infection with femoral venous catheters as compared to subclavian and internal jugular venous catheters: a systematic review of the literature and meta-analysis. Crit Care Med. 2012;40(8):2479-85. https://doi.org/10.1097/ CCM.0b013e318255d9bc

32. Costello JM, Graham DA, Morrow DF, Potter-Bynoe G, Sandora TJ, Laussen PC. Risk factors for central line-associated bloodstream infection in a pediatric cardiac intensive care unit. Pediatr Crit Care Med. 2009;10(4):453-9.

33. Loboguerrero GF. Infecciones asociadas a catéteres venosos centrales en la unidad de cuidado intensivo pediátrico. CES med. 2008;22:77-84.

34. Vilela R, Jácomo AD, Tresoldi AT. Risk factors for central venous catheter related infections in pediatric intensive care. Clinics. 2007;62:537-44.

35. Levy I, Bendet M, Samra Z, Shalit I, Katz J. Infectious complications of peripherally inserted central venous catheters in children. Pediatr Infect Dis J. 2010;29:426-9.

36. Warren DK, Quadir WW, Hollenbeak CS, Elward AM, Cox MJ, Fraser VJ. Attributable cost of catheter-associated bloodstream infections among intensive care patients in a nonteaching hospital. Crit Care Med. 2006;34:2084-9.

37. Shehab N, Patel PR, Srinivasan A, Budnitz DS. Emergency department visits for antibiotic-associated adverse events. Clin Infect Dis. 2008:47:735-43. https://doi.org/10.1086/591126. 
38. Hoeboer SH, van der Geest PJ, Nieboer D, Groeneveld AB. The diagnostic accuracy of procalcitonin for bacteraemia: a systematic review and metaanalysis. Clin Microbiol Infect. 2015;21(5):474-81. https://doi.org/10.1016/j. cmi.2014.12.026 Epub 2015 Jan 14.

39. Markus B, Peter AW. The inflammatory response in sepsis. Trends Immunol. 2013;34(3):129-36

\section{Publisher's Note}

Springer Nature remains neutral with regard to jurisdictional claims in published maps and institutional affiliations.

Ready to submit your research? Choose BMC and benefit from:

- fast, convenient online submission

- thorough peer review by experienced researchers in your field

- rapid publication on acceptance

- support for research data, including large and complex data types

- gold Open Access which fosters wider collaboration and increased citations

- maximum visibility for your research: over $100 \mathrm{M}$ website views per year

At BMC, research is always in progress.

Learn more biomedcentral.com/submissions 